\title{
PROPIEDADES PSICOMÉTRICAS DEL CUESTIONARIO DE NECESIDADES DE FAMILIARES DE PACIENTES HOSPITALIZADOS EN UNIDADES CRÍTICAS, VERSIÓN ABREVIADA
}

\author{
PSYCHOMETRIC PROPERTIES OF THE QUESTIONNAIRE \\ FOR NEEDS OF FAMILY MEMBERS OF PATIENTS HOSPITALIZED \\ IN CRITICAL UNITS, ABBREVIATED VERSION
}

\author{
Lidia Romina Villalobos Aburto * \\ Ximena Odette Osorio Spuler ${ }^{*}$ \\ José Luis VALDEbenito SANTANA ${ }^{* * *}$
}

\begin{abstract}
RESUMEN
El ingreso de una persona a una Unidad de Paciente Crítico genera efectos en su círculo familiar más cercano, siendo el acceso a la información y trato empático algunas de las necesidades de los familiares de los pacientes hospitalizados. El Critical Care Family Needs Inventory (CCFNI), versión breve, permite evaluar la satisfacción de necesidades de los familiares de pacientes hospitalizados en estas unidades y se considera una herramienta útil para obtener una visión de este grupo. Objetivo: Describir la adaptación cultural y evaluar las propiedades psicométricas del CCFNI, versión breve, en familiares de pacientes hospitalizados en Unidades de Cuidados Intensivos del Hospital Dr. Hernán Henríquez Aravena, Temuco, Chile. Material y método: Diseño de corte transversal. Se realizó la adaptación cultural del instrumento y luego se aplicó a 77 familiares, posterior a la firma del consentimiento informado. Se determinó validez de constructo y confiabilidad del instrumento. Resultados: Fueron excluidos los ítems 11 y 14. El análisis factorial exploratorio evidenció cuatro componentes cuyos alfa de Cronbach fueron 0,$7 ; 0,7 ; 0,6$ y 0,3. Conclusión: La traducción y adaptación realizada debe continuar su estudio psicométrico incorporando un número mayor de familiares de pacientes hospitalizados para confirmar la exclusión de los ítems y comprobar su estructura multidimensional. Este instrumento constituye una herramienta para valorar las necesidades de familiares de pacientes hospitalizados en Unidades Críticas.
\end{abstract}

Palabras clave: Validez de las pruebas, enfermería de cuidados críticos, Unidades de Cuidados Intensivos, necesidades y demandas de servicios de salud.

\begin{abstract}
The admission of a patient to the Intensive Care Unit has an effect on its inner family circle, the access to information and an empathic treatment being some of the needs of the relatives of the patient in this unit. The short version of the Critical Care Family Needs Inventory (CCFNI) allows to evaluate the satisfaction of those needs by the relatives of the patients in this units and it is considered a useful tool to obtain an overview of this group. Objective: To describe the cultural adaptations and to evaluate the psychometric properties of the short

\footnotetext{
* Enfermera, Magíster en Enfermería de la Universidad de La Frontera, docente Universidad Mayor, Temuco, Chile. Email: lidiavillalobos@gmail.com

${ }^{* *}$ Enfermera, Magíster en Enfermería de la Universidad de Concepción, Doctora $\odot$ de la Universidad Andrés Bello, académica Departamento de Enfermería, Universidad de La Frontera, Temuco. Chile. Email: ximena.osorio@ufrontera.cl

${ }^{* * *}$ Nutricionista, Universidad Autónoma, Temuco. Académico en la Universidad de Magallanes, Punta Arenas, Chile. Email: jvaldebenito.santana@gmail.com
} 
version of the CCFNI in hospitalized patients' relatives in Dr. Hernán Henríquez Aravena’s Hospital, Temuco, Chile. Method: This research uses a cross-sectional survey design. The tool was first culturally adapted and then applied to 77 relatives during the time span of five months, after a written consent was provided. Validity of the construct and reliability of the tool are determined. Results: Items 11 and 14 were excluded. The exploratory factorial analysis showed four components whose Alphas of Cronbach were 0.7; 0.7; 0.6 and 0.3. Conclusion: The translation and adapted version applied must continue its psychometric study incorporating a bigger number of relatives of hospitalized patients to confirm the exclusion of items and to check its multidimensional structure. This instrument is a tool to value the needs of relatives of patients hospitalized in Critical Units.

Key words: Validity of the tests, critical care nursing, Intensive Care Units, health services needs and demands.

Fecha recepción: 24/11/16 Fecha aceptación: 22/12/17

\section{INTRODUCCIÓN}

La hospitalización de una persona en una Unidad de Cuidados Intensivos produce una serie de necesidades en sus familiares; ellos presentan temor por la vida de su familiar hospitalizado, requieren información, esperan expresar sus propios sentimientos (1) y un trato empático del personal (2).

La revisión de la literatura da cuenta de varios estudios a nivel internacional donde se ha validado el Critical Care Family Needs Inventory (CCFNI) (3-6), destacando el estudio de Leske, realizado durante los años 1980-1988 en los Estados Unidos (3), debido a que la muestra la constituyeron 677 personas de 21 estados. En este estudio se determinó la validez y fiabilidad del cuestionario, identificándose cinco dimensiones en las necesidades de los familiares: Soporte, Confort, Información, Proximidad y Seguridad.

En Turquía, el año 2015, se tradujo, adaptó y validó el CCFNI en 191 familiares de pacientes hospitalizados en unidades críticas, obteniendo una consistencia interna, medida a través del alfa de Cronbach, de 0,93 para toda la escala; se realizó validez de contenido mediante el análisis por expertos y de constructo mediante el análisis factorial exploratorio (4).

El año 2014 se validó el CCFNI en Teherán Irán, aplicando el instrumento a 720 familias de pacientes hospitalizados en Unidad de Cuidados Intensivos y salas de cuidados generales, obteniendo un coeficiente alfa de Cronbach para todo el instrumento de 0.89 (5).

En Brasil el año 2004 (6) investigadores realizaron la "validación de criterio" de este instrumento, en un estudio que contó con la participación de 52 familiares de pacientes hospitalizados en UTI, utilizando métodos psicofísicos de estimación de magnitud. Con esto pudieron concluir que la escala es válida (coeficiente de correlación de Pearson de $r=0,97$ ), y evidenciaron que los resultados tenían una concordancia significativa entre los participantes.

Además, es trascendente señalar que el CCFNI no sólo se ha aplicado en familiares de pacientes adultos, sino también en el área neonatal. Es así como el estudio realizado en Unidades de Terapia Intensiva Neonatal el año 2010 en Brasil (7) presentó una confiabilidad de 0,83 , siendo las necesidades relacionadas con seguridad, información y proximidad de mayor importancia.

El año 1998 Johnson et al. realizaron la validación de la versión breve del CCFNI, para lo cual desarrollaron un análisis factorial con 12 ítems, e identificaron 4 componentes: actitud, comunicación, consuelo y aislamiento (2).

Con respecto a la versión abreviada del cuestionario, el año 2011 se tradujo y adaptó a población española, aplicándose a 55 familiares de pacientes ingresados en una UCI de 
Castellón (8). Para ello se utilizó la adaptación de Molter del cuestionario original (9). El CCFNI (versión breve) mostró una buena consistencia interna del cuestionario global, con un alfa de 0,655 y obtuvo una estructura de cuatro componentes que corresponden a: Atención Médica al paciente, Atención Personal al familiar, Información y Comunicación médico-paciente y Posibles mejoras percibidas (8).

En Chile existen limitadas publicaciones que analizan las necesidades de los familiares de pacientes hospitalizados en UCI/UTI y no se dispone de ningún instrumento validado para medirlas, por lo que se genera un vacío de información y posterga el diseño de planes de mejora de la calidad de la atención hacia este grupo particular de usuarios del sistema de salud (10).

Objetivo general: Describir la adaptación cultural y evaluar las propiedades psicométricas de la versión en español-chileno del Critical Care Family Needs Inventory (CCFNI), versión breve, en familiares de pacientes hospitalizados en unidades críticas de adulto, del Hospital Dr. Hernán Henríquez Aravena de Temuco, durante el primer semestre de 2015.

Objetivos específicos: 1. Adaptar el cuestionario cultural e idiomáticamente y 2 . Determinar las propiedades psicométricas iniciales (validez y confiabilidad) del CCFNI, versión breve.

\section{MATERIAL Y MÉTODO}

Esta investigación se enmarca en el área de estudios psicométricos de instrumentos de medición y se basa en la aplicación de pruebas estadísticas, con carácter objetivo, metódico y comprobable (11) para validez de constructo y confiabilidad del Critical Care Family Needs Inventory (CCFNI), versión breve.
El CCFNI, versión breve, contiene 14 ítems, a diferencia del original que consta de 45. Sus respuestas son en escala tipo Likert, con rango $1-4$ ( $1=$ Siempre; $2=$ A veces; $3=$ Nunca; $4=$ No responde). Se decidió validar la versión breve debido a que los familiares de los pacientes quieren estar el mayor tiempo posible al lado de su ser querido (1) y se encuentran con apremio emocional y angustia (10).

El universo correspondió a familiares de pacientes ingresados a UCI y UTI adultos, del Hospital Dr. Hernán Henríquez Aravena de Temuco de alta complejidad, del sur de Chile, en el período enero a junio de 2015, que cumplieron con los criterios de inclusión. La muestra no probabilística, intencionada, se calculó en función del instrumento, mediante método referido por Streiner y Norman (12), multiplicando el número de preguntas del cuestionario CCFNI versión breve por la constante sugerida por Streiner y Norman (3-5 aplicaciones por pregunta), obteniendo como resultado mínimo sugerido 70 aplicaciones.

Los criterios de inclusión considerados para los participantes del estudio fueron: ser familiar directo con edad superior a 18 años del paciente hospitalizado, por cualquier causa médico-quirúrgica y con un tiempo de estadía mínimo de 48 h y aceptación y firma del Consentimiento Informado (C.I.), previo a la aplicación del instrumento recolector de datos. Los criterios de exclusión fueron: que el familiar del paciente hospitalizado se encontrara en un estado emocional que lo inhabilite para participar del estudio y/o que no tenga la capacidad de comprensión para contestar el cuestionario.

Se inició el trabajo con la traducción al español-chileno de la versión breve del CCFNI realizada por una persona bilingüe con lengua española de origen y experiencia en literatura científica del ámbito sanitario (traducción directa). La traducción fue discutida y adaptada por la investigadora principal y dos docentes universitarias expertas en in- 
vestigación en salud, quienes sugirieron algunas modificaciones, que fueron incorporadas. Posteriormente se realizó la validez de fachada, presentando el instrumento a tres enfermeras expertas en Cuidados Intensivos adulto y a las dos docentes antes mencionadas, quienes agregaron nuevas sugerencias de redacción y manifestaron su aprobación.

Luego de generada la versión del instrumento en español, adaptado a la población chilena, se aplicó a un grupo de familiares de pacientes hospitalizados en UTI/UCI (20 personas) durante los períodos de horarios de visita (13.00 a 14.00 y 19.00 a 20.00 h), en la sala de espera de UCI/UTI. En esta fase se realizó análisis de la comprensión de las preguntas por parte de los participantes, sin haberse generado modificaciones del mismo.

La versión final fue aplicada a un total de 77 familiares de pacientes hospitalizados en UCI-UTI del Hospital Dr. Hernán Henríquez Aravena, de los cuales, un 55,8\% pertenecían al servicio clínico UTI y un $44,2 \%$ a UCI. La recolección de los datos fue realizada por los investigadores del estudio y un grupo de personas relacionadas con el área de salud, previamente capacitadas y supervisadas.

Los datos fueron ingresados a una hoja de planilla Excel, posterior a esto se realizó su traslado al programa estadístico SPSS versión 22, donde se estimó confiabilidad a través de alfa de Cronbach, pruebas de Kaiser-Meier-Olkin (KMO) (13), que contrasta si las correlaciones parciales entre las variables son suficientemente pequeñas, permitiendo comparar los coeficientes de correlación observados con los coeficientes de correlación parcial, lo que corresponde a un análisis factorial exploratorio (14); este último se usa para obtener evidencias de las dimensiones subyacentes (componentes) que están presentes en el instrumento y que deberían corresponder, en teoría, al constructo que se quiere medir (15). En esta investigación no se realizó un análisis factorial comprobatorio, ya que el ajuste del modelo hace referencia a qué tan bien el modelo de fac- tores propuesto se ajusta a las correlaciones entre las variables del conjunto de datos (16, 17).

Para la realización del presente estudio se dispuso de los permisos correspondientes a una investigación donde participan seres humanos, contándose con la autorización del Comité de ética del Servicio de Salud Araucanía Sur, del director (Resol. Exenta $N^{\circ}$ 620, del 9 de diciembre de 2014) y jefatura de UCI/UTI del Hospital Hernán Henríquez Aravena.

\section{RESULTADOS}

Respecto a los datos sociodemográficos de la muestra, se obtuvo que el 41,6\% de familiares correspondía a hermanos/as de los pacientes. Un $66,2 \%$ de ellos eran de sexo masculino y el 57,1\% habían cursado enseñanza media. El $16,9 \%$ pertenecían a la etnia mapuche, mientras que el 75,3\% de la muestra residían en sector urbano (Tabla 1). En relación a la edad de los familiares, se obtuvo un promedio de 45,8 años (DS 15,6), con edades comprendidas entre 19 y 90 años y respecto de los días de hospitalización de los pacientes, se promediaron en 12,6 (DS 14,5) con un mínimo de 2 y un máximo de 60 días.

En relación a los ítems del cuestionario, se encontró que un $88 \%$ de los familiares creen que "siempre" se les está entregando el mejor cuidado posible a su familiar hospitalizado. Un 68,8 y $71,4 \%$ de los familiares afirman que "siempre" han entendido las explicaciones con respecto al estado de salud del paciente y que les han entregado información honesta respecto al estado de su familiar, respectivamente. Un $83,1 \%$ de la muestra afirma que "siempre" están muy satisfechos con los cuidados de Enfermería que recibe su familiar (Tabla 2). Contrasta a los resultados anteriores, un $61 \%$ de los familiares que afirman que "nunca" ninguno de los miembros del equipo de salud ha manifestado interés 
Tabla 1. Caracterización sociodemográfica de los familiares de pacientes hospitalizados en UCI-UTI, Hospital Dr. Hernán Henríquez Aravena. Temuco. 2015.

\begin{tabular}{llrr}
\hline Variables & & F & $\%$ \\
\hline Tipo de parentesco & Madre & 7 & 9,1 \\
& Padre & 2 & 2,6 \\
& Esposo/a & 9 & 11,7 \\
& Hijo/a & 26 & 33,7 \\
& Hermano/a & 32 & 41,6 \\
Sexo & Pareja/conviviente & 1 & 1,3 \\
& Masculino & 51 & 66,2 \\
Escolaridad & Femenino & 26 & 33,8 \\
& Nivel Básico & 18 & 23,4 \\
& Enseñanza media & 44 & 57,1 \\
& Enseñanza superior & 15 & 19,5 \\
Etnia & Otra & 0 & 0 \\
& Mapuche & 13 & 16,9 \\
& No Mapuche & 44 & 57,1 \\
& Otra* & 1 & 1,3 \\
Residencia & No Informado & 19 & 24,7 \\
& Urbano & 58 & 75,3 \\
\hline
\end{tabular}

* Etnia: Pehuenche.

Tabla 2. Distribución de frecuencia por ítems del Cuestionario de Necesidades de Familiares Hospitalizados en UCI-UTI, según respuesta, del Hospital Dr. Hernán Henríquez Aravena, Temuco. 2015.

\begin{tabular}{|c|c|c|c|c|c|c|c|c|}
\hline \multirow[t]{2}{*}{ Items } & \multicolumn{2}{|c|}{ Siempre } & \multicolumn{2}{|c|}{ A veces } & \multicolumn{2}{|c|}{ Nunca } & \multicolumn{2}{|c|}{ No responde } \\
\hline & f & $\%$ & f & $\%$ & f & $\%$ & f & $\%$ \\
\hline $\begin{array}{l}\text { ¿Cree usted que se está entregando el mejor cuidado } \\
\text { posible a su familiar hospitalizado? }\end{array}$ & 68 & 88,3 & 7 & 9,1 & 2 & 2,6 & 0 & 0 \\
\hline $\begin{array}{l}\text { ¿Cree usted que el personal del hospital se preocupa } \\
\text { por su familiar? }\end{array}$ & 61 & 79,2 & 14 & 18,2 & 2 & 2,6 & 0 & 0 \\
\hline $\begin{array}{l}\text { ¿Ha entendido las explicaciones que le han dado res- } \\
\text { pecto al estado de salud de su familiar? }\end{array}$ & 53 & 68,8 & 14 & 18,2 & 8 & 10,4 & 2 & 2,6 \\
\hline $\begin{array}{l}\text { ¿Cree usted que se le ha entregado información ho- } \\
\text { nesta acerca del estado de salud y pronóstico de su } \\
\text { familiar hospitalizado? }\end{array}$ & 55 & 71,4 & 11 & 14,3 & 10 & 13 & 1 & 1,3 \\
\hline $\begin{array}{l}\text { ¿Entiende usted lo que está ocurriendo con su fa- } \\
\text { miliar y por qué está hospitalizado (enfermedad y } \\
\text { tratamiento)? }\end{array}$ & 62 & 80,5 & 7 & 9,1 & 7 & 9,1 & 1 & 1,3 \\
\hline $\begin{array}{l}\text { ¿Las personas del equipo de salud que atienden a su } \\
\text { familiar son atentos y respetuosos con usted? }\end{array}$ & 68 & 88,3 & 7 & 9,1 & 1 & 1,3 & 1 & 1,3 \\
\hline
\end{tabular}


¿Ha mostrado alguno de los miembros del equipo de salud interés en cómo está usted?

19

¿Cree usted que alguien del hospital lo llamará a la casa en caso de algún cambio importante en la salud de su familiar?

¿Le ha explicado el personal del hospital cómo funcionan los equipos y máquinas que están siendo usadas para tratar a su familiar?

¿Está usted muy satisfecho con el cuidado de Enfermería (enfermera y técnicos paramédicos) que recibe su familiar?

¿Hay algunas cosas en el tratamiento médico que su familiar recibe que usted cree que podrían ser mejores?

(1)

¿Se siente cómodo al visitar a su familiar en la Unidad de Cuidados Intensivos- UTI?

¿Es cómoda la sala de espera?

¿Se siente solo/a y aislado/a en el área de espera?

en cómo están ellos y otro $61 \%$ de los encuestados, que señalan que "nunca" el personal de salud les ha explicado cómo funcionan los equipos y máquinas que están siendo usadas para tratar a su familiar.

A través del análisis de consistencia interna del cuestionario se determinó un alfa de Cronbach de 0,69, considerado como un valor "débil", dado que se determinó primeramente la confirmabilidad global del instrumento, pues el alfa de Cronbach asume que los ítems (medidos en escala tipo Likert) miden un mismo constructo y que están altamente correlacionados, para luego seguir con los siguientes análisis $(18,19)$.

Inicialmente, se estudió el cuestionario original con sus 14 ítems y se realizó el análisis de confiabilidad de alfa de Cronbach de éste. Se observó que al eliminar la pregunta 11 (“¿Hay algunas cosas del tratamiento médico que su familiar recibe que usted cree que podrían ser mejores?") del cuestionario, la confiabilidad ascendía a 0,662 (Tabla 3). Posterior a la eliminación del ítem $\mathrm{N}^{\circ} 11$, se realizó un nuevo análisis, que evidenció que al suprimir la pregunta número 14 (“ ¿Se siente solo en la sala de espera?") la confiabilidad del instrumento subía de 0,662 a 0,689 (Tabla 3).

Posteriormente se realizó el análisis factorial, efectuándose las pruebas KMO de 0,65, considerado aceptable, y la evaluación de esferidad de Bartlett $c^{2}(77)=223,610$, valor $\mathrm{p}=0,000$, por lo que se concluyó que era adecuado realizar un análisis factorial.

Se utilizó el método de extracción de componentes principales con rotación Varimax, revelando que los primeros 4 ítems del cuestionario explicaron el 63,46\% de la varianza total. Los resultados del análisis factorial se observan en la Tabla 4.

Con la finalidad de agrupar los ítems del cuestionario en factores (Tabla 5), se realizó un análisis de selección de las cargas más altas, donde destacó que: el factor 1 se conforma por las preguntas 1, 3, 4 y 5, y está relacionado con la información que percibe recibir el familiar, por lo que se denominó "comunicación" y presentó un alfa de 0,697 considerado, como "débil". El factor 2 se compone por las preguntas 2,8 y 10 , que evalúan percepción de la atención entregada 
por el equipo de salud, por lo que se denominó "atención del equipo" y evidenció un alfa de 0,713 catalogado como "aceptable. El factor 3, que incluye las preguntas 9, 12 y 13, se relaciona con la satisfacción de los aspectos que podrían ser mejorados, relacionados con la atención de salud y comodidad que se entrega al familiar, por lo que se le señaló como "posibles mejoras"; éste mostró un alfa de 0,596 , considerado como "pobre"; y por último el factor 4 , que contiene los ítems 6 y 7, se relaciona con la percepción de interés por parte del equipo de salud y se le nombró como "consuelo", arrojando un alfa de 0,335 y demostrando un nivel de fiabilidad "no aceptable".

La Tabla 6 presenta la comparación entre los estudios de Johnson et al. (2), Gómez et al. (8) y la agrupación de ítems a partir del análisis factorial propuesto por los investigadores.

Tabla 3. Alfa de Cronbach si se suprime ítems $\mathrm{N}^{\text {os }} 11$ y 14 del Cuestionario de Necesidades de Familiares Hospitalizados en UCI-UTI, versión breve, del Hospital Dr. Hernán Henríquez Aravena, Temuco. 2015.

\section{Ítem del Cuestionario}

\section{Alfa de Cronbach Alfa de Cronbach \\ si el elemento 11 si el elemento 14 \\ se ha suprimido se ha suprimido}

1 ¿Cree usted que se está entregando el mejor cuidado posible a su familiar hospitalizado?

\begin{tabular}{|c|c|}
\hline 0,525 & 0,636 \\
\hline 0,525 & 0,637 \\
\hline 0,511 & 0,632 \\
\hline 0,512 & 0,629 \\
\hline 0,549 & 0,663 \\
\hline 0,544 & 0,654 \\
\hline 0,53 & 0,646 \\
\hline 0,524 & 0,642 \\
\hline 0,496 & 0,633 \\
\hline 0,51 & 0,628 \\
\hline 0,662 & - \\
\hline 0,484 & 0,615 \\
\hline 0,539 & 0,660 \\
\hline 0,594 & 0,689 \\
\hline
\end{tabular}

14 ¿Se siente solo/a y aislado/a en el área de espera? 
Tabla 4. Análisis factorial exploratorio del Cuestionario de Necesidades de Familiares Hospitalizados en UCI-UTI, versión breve, del Hospital Dr. Hernán Henríquez Aravena. Temuco, 2015.

\begin{tabular}{|c|c|c|c|c|c|c|}
\hline \multirow[b]{2}{*}{ Componente } & \multicolumn{3}{|c|}{ Autovalores iniciales } & \multicolumn{3}{|c|}{ Sumas de extracción de cargas al cuadrado } \\
\hline & Total & $\begin{array}{c}\% \text { de } \\
\text { varianza }\end{array}$ & $\begin{array}{c}\% \\
\text { acumulado }\end{array}$ & Total & $\begin{array}{c}\% \\
\text { de varianza } \\
\end{array}$ & $\begin{array}{c}\% \\
\text { acumulado }\end{array}$ \\
\hline 1 & 3,058 & 25,485 & 25,485 & 3,058 & 25,485 & 25,485 \\
\hline 2 & 2,072 & 17,263 & 42,748 & 2,072 & 17,263 & 42,748 \\
\hline 3 & 1,427 & 11,894 & 54,642 & 1,427 & 11,894 & 54,642 \\
\hline 4 & 1,059 & 8,822 & 63,464 & 1,059 & 8,822 & 63,464 \\
\hline 5 & 0,966 & 8,047 & 71,511 & & & \\
\hline 6 & 0,725 & 6,041 & 77,552 & & & \\
\hline 7 & 0,645 & 5,377 & 82,929 & & & \\
\hline 8 & 0,499 & 4,162 & 87,091 & & & \\
\hline 9 & 0,48 & 4,001 & 91,092 & & & \\
\hline 10 & 0,452 & 3,767 & 94,859 & & & \\
\hline 11 & 0,363 & 3,025 & 97,884 & & & \\
\hline 12 & 0,254 & 2,116 & 100 & & & \\
\hline
\end{tabular}

Tabla 5. Matriz de componentes rotados de la escala Cuestionario de Necesidades de Familiares Hospitalizados en UCI-UTI, versión breve, del Hospital Dr. Hernán Henríquez Aravena, Temuco, 2015.

Ítems

Componente

$\begin{array}{llll}1 & 2 & 3 & 4\end{array}$

1. ¿Cree usted que se está entregando el mejor cuidado posible a su familiar hospitalizado?

2. ¿Cree usted que el personal del hospital se preocupa por su familiar?

3. ¿Ha entendido las explicaciones que le han dado respecto al estado de salud de su familiar?

\begin{tabular}{cccc}
$\mathbf{0 , 6 9 9}$ & 0,406 & 0,025 & $-0,129$ \\
$-0,014$ & $\mathbf{0 , 8 4 8}$ & 0,148 & 0,053 \\
$\mathbf{0 , 7 6 7}$ & $-0,087$ & 0,152 & 0,144 \\
$\mathbf{0 , 7 7 9}$ & 0,11 & 0,138 & $-0,154$ \\
$\mathbf{0 , 6 1 9}$ & $-0,075$ & $-0,198$ & 0,288 \\
$-0,108$ & 0,124 & 0,092 & $\mathbf{0 , 8 8 5}$ \\
0,179 & 0,052 & 0,27 & $\mathbf{0 , 5 3}$ \\
$-0,082$ & $\mathbf{0 , 7 8 6}$ & 0,228 & 0,011 \\
0,249 & 0,045 & $\mathbf{0 , 5 2 6}$ & 0,123 \\
& & & \\
0,349 & $\mathbf{0 , 7 5 8}$ & $-0,055$ & 0,198 \\
0,122 & 0,193 & $\mathbf{0 , 7 8 9}$ & 0,133 \\
$-0,254$ & 0,095 & $\mathbf{0 , 8}$ & 0,076 \\
\hline
\end{tabular}




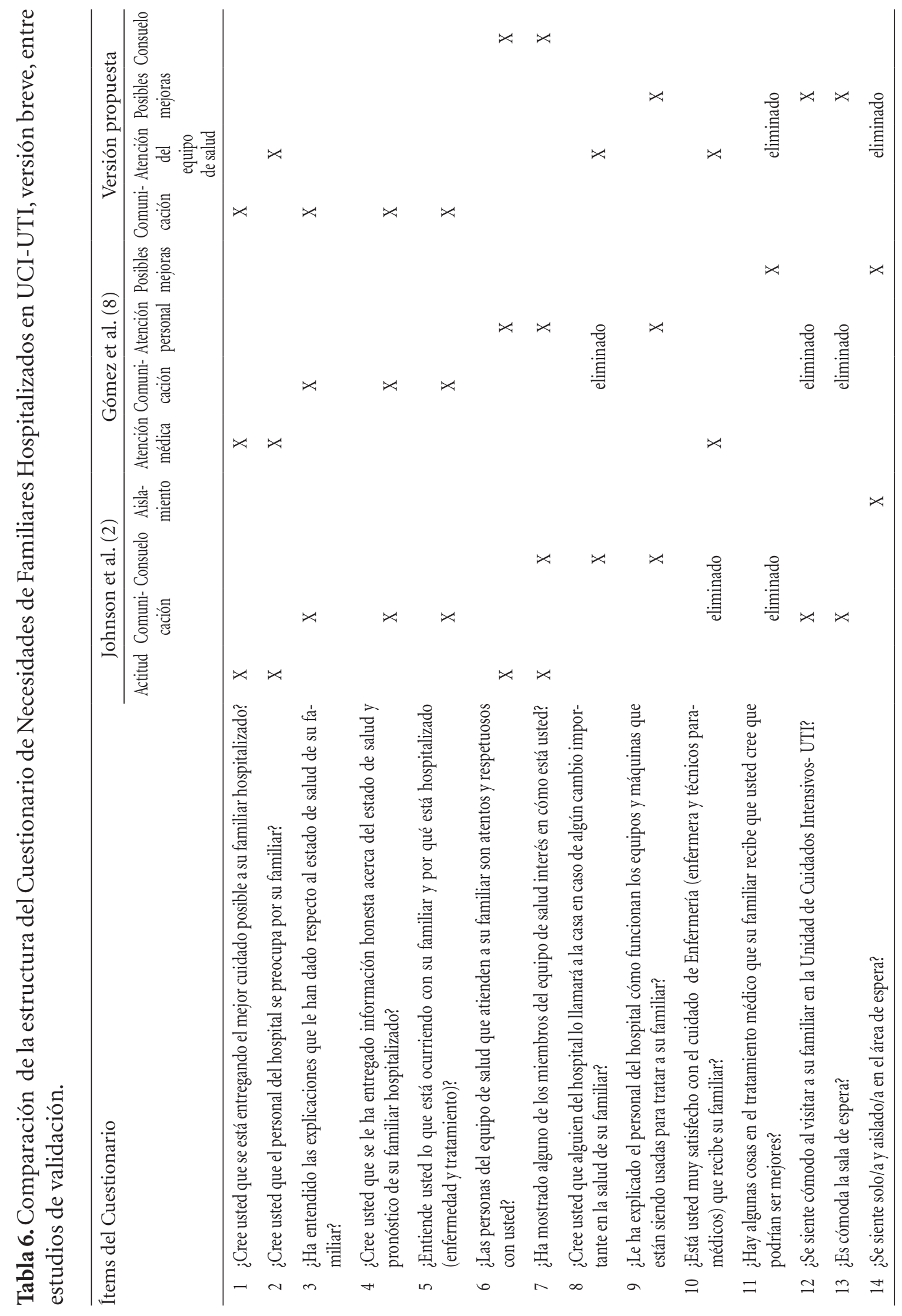




\section{DISCUSIÓN Y CONCLUSIÓN}

Valorar las necesidades de los familiares de pacientes en unidades críticas y apreciar la capacidad de lograr un adecuado abordaje de éstas, por parte del equipo de salud, es un proceso complejo, tanto por su amplitud como por su carácter subjetivo. Por este motivo se han diseñado instrumentos que ayudan a obtener una visión más objetiva y concreta de las mismas. A su vez, se ha evidenciado que la determinación de validez y confiabilidad de distintos instrumentos de medición constituye un aporte empírico para colaborar en la orientación de la Gestión del Cuidado, con una mirada más humanizada (11).

Al realizar la comparación del presente estudio con la validación del CCFNI versión breve en inglés (2) y española (8), se puede apreciar que la muestra utilizada en este estudio $(n=77)$ es menor que la de Johnson et al. (2) $(n=99)$, pero mayor que la de Gómez et al. (8) $(n=55)$. En relación a este punto, se aprecia que tanto en el estudio español (8) como en el actual hubo dificultad para obtener la totalidad de la muestra estimada.

Con respecto a los valores del índice de confiabilidad de este estudio (alfa de Cronbach $=0,69)$, se aprecia que fueron mayores que el de Gómez et al. $(0,655)$, pero menores al de Johnson et al. $(0,76)$, resultado que coincide de manera directamente proporcional al número de participantes de cada estudio.

Los resultados del análisis factorial exploratorio muestran una estructura de cuatro componentes, denominados comunicación, atención del equipo de salud, consuelo y posibles mejoras, que guardan relación con lo evidenciado en la versión breve de Johnson et al. (2) y Gómez et al. (8). En la versión de Molter (9) se aislaron cinco factores, los que fueron señalados como: la necesidad de apoyo, confort, información, proximidad y seguridad.

Para realizar una comparación del pre- sente estudio con otros similares, se diseñó la Tabla 6, donde se aprecia que el ítem 1 es similar en las tres investigaciones, siendo denominado en el presente estudio "comunicación", "actitud" en el de Johnson et al. (2) y “atención médica” en el de Gómez et al. (8).

El ítem 9 coincide en el mismo factor en los 3 estudios; en el trabajo de Johnson et al. (2) se le asigna el nombre de "consuelo", en el de Gómez et al. (8) "atención personal” y en el actual "posibles mejoras”. Es importante recalcar que los componentes resultantes del análisis factorial en los tres estudios se denominan de manera diferente. Los investigadores consideraron que los conceptos utilizados en las otras investigaciones presentaban una acepción diferente a la realidad local; lo que responde, en parte, a las adaptaciones culturales propias de cada investigación.

En referencia a la eliminación de los ítems, es importante mencionar que el ítem 11 fue eliminado del estudio de Johnson et al. (2), y del actual, mientras que en el de Gómez et al. (8) pertenecía al factor 4, denominado “posibles mejoras". Además el ítem número 14 fue eliminado de la presente investigación, mientras que en el estudio de Johnson et al. (2) y de Gómez et al. (8) pertenecía al factor 4, denominado "aislamiento" y "posibles mejoras", respectivamente. Respecto a ello, la literatura describe que es posible observar un incremento en la consistencia con la reducción en el número de ítems cuando la o las preguntas eliminadas son de poca calidad y exploran pobremente el constructo. Además se menciona que el impacto en la confiabilidad es más marcado con la reducción del número de ítems si se ensaya con una muestra relativamente pequeña (20), como es en este caso en particular.

Al observar la consistencia interna de los factores del presente estudio y realizar comparación con las otras publicaciones, se aprecia que el factor 1 presenta un alfa inferior al de Johnson et al. (2), pero superior al de Gómez et al. (8). De forma distinta, el factor 2, llamado en este trabajo "atención del 
equipo de salud", presenta un alfa superior al mismo de Johnson et al. (2) y Gómez et al. (8) ("comunicación"), con valores de 0,713 , 0,696 y 0,603 , respectivamente. Además se aprecia que el 3 , denominado "posibles mejoras", presenta un alfa superior al de Johnson et al. $(2)(0,552)$, pero inferior al de Gómez et al. (8) $(0,605)$. Un comportamiento muy diferente ocurre con el 4 , señalado en este estudio como "consuelo", el que presenta un alfa que es marcadamente inferior al estudio de Gómez et al. (8) $(0,641)$. No fue posible realizar la comparación de este punto con la validación de Johnson et al. (2), ya que no se publicó esa información puntual.

Es preciso mencionar que dentro de las limitaciones del estudio, la principal fue la posibilidad de obtención del tamaño muestral $(n=77)$, que se explica en parte por el momento emocional en que se encuentran los familiares de pacientes hospitalizados en situación crítica de salud, aspecto considerado en los criterios de exclusión, además de la disposición y las características del lugar (sala de espera pequeña), lo que hace muy dificultosa la obtención de la muestra. Este fenómeno se describe de manera similar en las investigaciones que abordan a familiares de pacientes en UCI-UTI $(9,21)$.

Por otra parte, cabe mencionar que no se realizó la retrotraducción de la versión traducida al español-chileno por un hablante nativo de inglés, ni su posterior comparación con la versión original.

De los resultados obtenidos se puede concluir que la traducción y adaptación realizada debe continuar su estudio psicométrico, incorporando un número mayor de familiares de pacientes hospitalizados para confirmar la exclusión de los ítems 11 y 14 y comprobar su estructura multidimensional.

El disponer de un cuestionario validado en el país otorgará la posibilidad de aplicarlo en las distintas Unidades de Paciente Crítico, con la confianza de que entregará datos reales y comparables con parámetros nacionales y extranjeros, cuya visión será de utili- dad para proponer mejoras en la satisfacción de necesidades de los familiares de pacientes hospitalizados en estos servicios.

\section{REFERENCIAS}

1. Álvarez Y. Unidad de Cuidados Intensivos: Un campo inexplorado por el Psicólogo en Chile. Revista de Psicología. 1999; 1(1): 7-16.

2. Johnson D, Wilson M, Cavanaugh B, Bryden C, Gudmundson D, Moodley O. Measuring the ability to meet family needs in an intensive care unit. Crit Care Med. 1998; 26(2): 266-71.

3. Leske J. Internal psychometric properties of the Critical Care Family Needs Inventory. Heart Lung. 1991; 20(3): 236-44.

4. Büyükçoban S, Çiçeklioğlu M, Demiral $\mathrm{N}$, Civaner M. Adaptation of the Critical Care Family Need Inventory to the Turkish population and its psychometric properties. Peer] [Internet]. 2015 [citado 20 dic 2017]; 3:e1208. Disponible en https:// www.ncbi.nlm.nih.gov/pmc/articles/ PMC4563234/

5. Bandari R, Heravi-Karimooi M, Rejeh N, Montazeri A, Zayeri F, Mirmohammadkhani M, et al. Psychometric Properties of the Persian Version of the Critical Care Family Needs Inventory. J Nurs Res. 2014; 22(4): 259-267.

6. Bijttebier P, Delva D, Vanoost S, Bobbaers $\mathrm{H}$, Lauwers P, Vertommen H. Reliability and validity of the Critical Care Family Needs Inventory in a Dutch-speaking Belgian sample. Heart Lung. 2000; 29(4): 278-86.

7. Oliveira L, Ferreira R, Gasparino Renata. Necessidades de familiares de pacientes internados em unidade de terapia intensiva neonatal. Texto contexto-enferm. 2010; 19(4): 644-650.

8. Gómez S, Ballester R, Gil B. El Cuestionario de Necesidades de los Familiares de 
Pacientes de Cuidados Intensivos (CCF$\mathrm{NI})$ versión breve: adaptación y validación en población española. Anales Sis San Navarra. 2011; 34(3): 349-361.

9. Molter N. Needs of relatives of critically ill patients: a descriptive study. Heart Lung. 1979; 8(2): 332-9.

10. Ugarte S, Valenzuela J, Guerra A, Ramírez $\mathrm{M}$. Encuesta sobre grado de satisfacción y evaluación de calidad de atención por familiares de enfermos de UCI. Rev Chil Med Intensiv. 2003; 18(1): 34-38.

11. Rivas A. Rivas E, Bustos L. Validación de escala de valoración del dolor en recién nacidos en una unidad de Neonatología. Cienc enferm. 2012; XVIII(2): 93-99.

12. Streiner D, Norman G. Health Measurement Scales: a Practical Guide to their Development and Use. 3rd ed. Oxford: Oxford University Press; 2008. 431 p.

13. Ventura J, Caycho T. Análisis psicométrico de una escala de dependencia emocional en universitarios peruanos. Rev. Psicología. 2016; 25(1): 1-17.

14. Detrinidad E. Análisis Factorial Exploratorio y Confirmatorio aplicado al modelo de secularización propuesto por Inglehart-Norris. 2016. Estudio de caso España, Estados Unidos, Alemania, Holanda WSV. [Tesis de Magíster]. Departamento de Estadística e Investigación Operativa: Universidad de Granada; 2016. 81 p.

15. Tangarife L, Arias C. Construcción y validación de escalas de medición en salud: revisión de propiedades psicométricas. Archivos de Medicina [Internet]. 2015 [citado 20 dic 2017]; 11(3:1). Disponible en: http://www.archivosdemedicina.com/ medicina-de-familia/construccin-y-validacin-de-escalasde-medicin-en-salud-revisin-depropiedades-psicomtricas. pdf

16. Gaskin, J. Hapell B. On exploratory factor analysis: A review of recent evidence, an assessment of current practice, and recommendations for future use. J. Nurs Stude. 2014; 51(3): 511-521.

17. Sicilia A, Alías-García A, Ferriz R, Moreno-Murcia JA. Spanish adaptation and validation of the Exercise Addiction Inventory (EAI). Psicothema. 2013; 25(3): 377-383.

18. Carvajal A, Centeno C, Watson R, Martínez M, Sanz Rubiales Á. ¿Cómo validar un instrumento de medida de la salud? Anales Sis San Navarra. 2011; 34(1): 6372.

19. Polit D, Hungler B. Investigación científica en ciencias de la salud. Sexta ed. México: McGraw-Hill Interamericana; 2000. $750 \mathrm{p}$.

20. Campo A, Oviedo H. Propiedades Psicométricas de una Escala: la Consistencia Interna. Rev. Salud Pública (Bogotá) [Internet]. 2008 [citado 20 dic 2017]; 10(5): 831-839. Disponible en: http://www.redalyc.org/articulo.oa?id=42210515

21. Gómez P, Soriano J, Monsalve V, Andrés J de. Satisfacción con la Información: Posible Variable Interviniente en el Estado de Ánimo de Cuidadores Primarios de Pacientes Críticos. Clínica y Salud. 2009; 20(1): 91-105. 